\title{
A Comparative Analysis of Models Used to Evaluate the Cost- Effectiveness of Dabigatran Versus Warfarin for the Prevention of Stroke in Atrial Fibrillation
}

\author{
Sonja V. Sorensen $\cdot$ Siyang Peng $\cdot$ Brigitta U. Monz $\cdot$ \\ Carole Bradley-Kennedy $\cdot$ Anuraag R. Kansal
}

Published online: 25 April 2013

(C) The Author(s) 2013. This article is published with open access at Springerlink.com

\begin{abstract}
Background A number of models exploring the costeffectiveness of dabigatran versus warfarin for stroke prevention in atrial fibrillation have been published. These studies found dabigatran was generally cost-effective, considering well-accepted willingness-to-pay thresholds, but estimates of the incremental cost-effectiveness ratios (ICERs) varied, even in the same setting. The objective of this study was to compare the findings of the published economic models and identify key model features accounting for differences.

Methods All aspects of the economic evaluations were reviewed: model approach, inputs, and assumptions. A previously published model served as the reference model for comparisons of the selected studies in the US and UK settings. The reference model was adapted, wherever possible, using the inputs and key assumptions from each of the other published studies to determine if results could be reproduced in the reference model. Incremental total costs, incremental quality-adjusted life years (QALYs), and ICERs (cost per QALY) were compared between each study and the corresponding adapted reference model. The impact of each modified variable or assumption was tracked separately.
\end{abstract}

S. V. Sorensen $(\varangle) \cdot$ S. Peng · A. R. Kansal

United BioSource Corporation, 7101 Wisconsin Avenue,

Suite 600, Bethesda, MD 20814, USA

e-mail: Sonja.sorensen@unitedbiosource.com

B. U. Monz

Boehringer Ingelheim GmbH, Ingelheim am Rhein, Germany

C. Bradley-Kennedy

Boehringer Ingelheim (Canada) Ltd., Burlington, ON, Canada
Results The selected studies were in the US setting (2), the Canadian setting (1), and the UK setting (2). All models used the Randomized Evaluation of Long-Term Anticoagulation study (RE-LY) as the main source for clinical inputs, and all used a Markov modelling approach, except one that used discrete event simulation. The reference model had been published in the Canadian and UK settings. In the UK setting, the reference model reported an ICER of UK£4,831, whereas the other UK-based analysis reported an ICER of UK£23,082. When the reference model was modified to use the same population characteristics, cost inputs, and utility inputs, it reproduced the results of the other model (ICER UK£25,518) reasonably well. Key reasons for the different results between the two models were the assumptions on the event utility decrement and costs associated with intracranial haemorrhage, as well as the costs of warfarin monitoring and disability following events. In the US setting, the reference model produced an ICER similar to the ICER from one of the US models (US\$15,115/QALY versus US\$12,386/QALY, respectively) when modelling assumptions and input values were transferred into the reference model. Key differences in results could be explained by the population characteristics (age and baseline stroke risk), utility assigned to events and specific treatments, adjustment of stroke and intracranial haemorrhage risk over time, and treatment discontinuation and switching. The reference model was able to replicate the QALY results, but not the cost results, reported by the other US cost-effectiveness analysis. The parameters driving the QALY results were utility values by disability levels as well as utilities assigned to specific treatments, and event and background mortality rates.

Conclusions Despite differences in model designs and structures, it was mostly possible to replicate the results published by different authors and identify variables 
responsible for differences between ICERs using a reference model approach. This enables a better interpretation of published findings by focusing attention on the assumptions underlying the key model features accounting for differences.

\section{Key Points for Decision Makers}

- Several economic models have assessed the costeffectiveness of dabigatran for stroke prevention in atrial fibrillation patients in different settings. These models generally found dabigatran to be cost-effective, but the incremental cost-effectiveness ratios (ICERs) varied considerably between them.

- Although the published economic models all use the RE-LY trial to inform key clinical parameters, there were differences in the model assumptions, inputs, structures, and approaches. This study quantifies the differences that led to the different ICER estimates.

- This study assessed quantitatively why the published cost-effectiveness models differed in their overall results and identified the relevant model parameters. Decision makers are then better able to challenge the relevant parameters to arrive at consistent conclusions, even if provided with different model approaches.

\section{Introduction}

Atrial fibrillation (AF) is a common arrhythmia and the leading cause of stroke, an event with high human and economic burden. In the UK, $1.28 \%$ of the total population has AF [1]. In the USA, approximately 2.7 million people had AF in 2010, with a prevalence estimate of $3.8 \%$ in the 65 and older population, and rates are expected to increase [2]. The direct cost of AF-related stroke is up to UK£24,000 per patient in the UK [3], whereas in the USA, the estimated direct and indirect economic burden of allcause stroke was US\$34.3 billion in 2008 [4].

For AF patients at moderate to high risk of stroke, anticoagulation for the prevention of ischaemic stroke is recommended by treatment guidelines [5-7]. Two of the available therapies are warfarin, a vitamin $\mathrm{K}$ antagonist, and dabigatran etexilate (dabigatran), a direct thrombin inhibitor. On the basis of the benefits demonstrated in the Randomized Evaluation of Long-Term Anticoagulation Therapy (RE-LY) trial $[8,9]$, dabigatran has been recommended as a cost-effective treatment option in several countries [5, 10-12].

Through April 2012, seven economic evaluations have been published in scientific journals assessing dabigatran versus warfarin in the USA [13-15], the UK [16, 17],
Canada [18], and Denmark [19]. The findings of these models were consistent in indicating the cost-effectiveness of dabigatran $150 \mathrm{mg}$ bid (especially in the US setting where the $110 \mathrm{mg}$ bid dose is not available), or the "sequential" use of $110 \mathrm{mg}$ bid and $150 \mathrm{mg}$ bid use ( $150 \mathrm{mg}$ bid for patients under 80 years old, $110 \mathrm{mg}$ bid for patients at least 80 years old as per the European and Canadian labels) compared to warfarin versus conventional willingness-to-pay thresholds (US\$100,000/QALY [20]; UK£30,000/QALY [21]). The absolute values of the incremental cost-effectiveness ratios (ICERs) varied, however, even within a country setting (Table 1). All studies found that the ICERs for dabigatran varied with baseline stroke risk and the level of International Normalized Ratio (INR) control achieved in patients on warfarin, but the final conclusions regarding cost-effectiveness with respect to these factors, particularly INR control, varied.

The availability of different models developed to answer the same general question creates an opportunity to explore the modelling approaches and the assumptions made, to better understand the reasons for any differences in model outcomes [22]. In this study, we compare the findings of published economic models to a reference model. The reference model was adapted incrementally using the inputs and assumptions from each of the selected published models individually to allow a detailed understanding of the major determinants of model result differences. This approach provides more insight than a qualitative review and descriptive comparison of published models.

\section{Methods: Review of Studies}

\subsection{Selection of Relevant Studies}

The published cost-effectiveness studies considered in this study were identified using the MEDLINE electronic database (via PubMed). The search terms were "costeffectiveness" and "dabigatran" and "atrial fibrillation", and the search was limited to studies published in English through April 2012. The resulting abstracts were screened for papers reporting original cost-effectiveness models, which yielded a total of seven studies [13-19]. Only the models that examined the same patient populations and the same overall research question underwent a detailed comparison.

The study by Kamel et al. [14] was excluded from this analysis because the patient population reflected only a subgroup of patients who had experienced a prior stroke or TIA. The study by Langkilde et al. [19] was also excluded as it was based on the model used in the current study as the reference model and no other Danish-setting costeffectiveness analysis was available for comparison. 


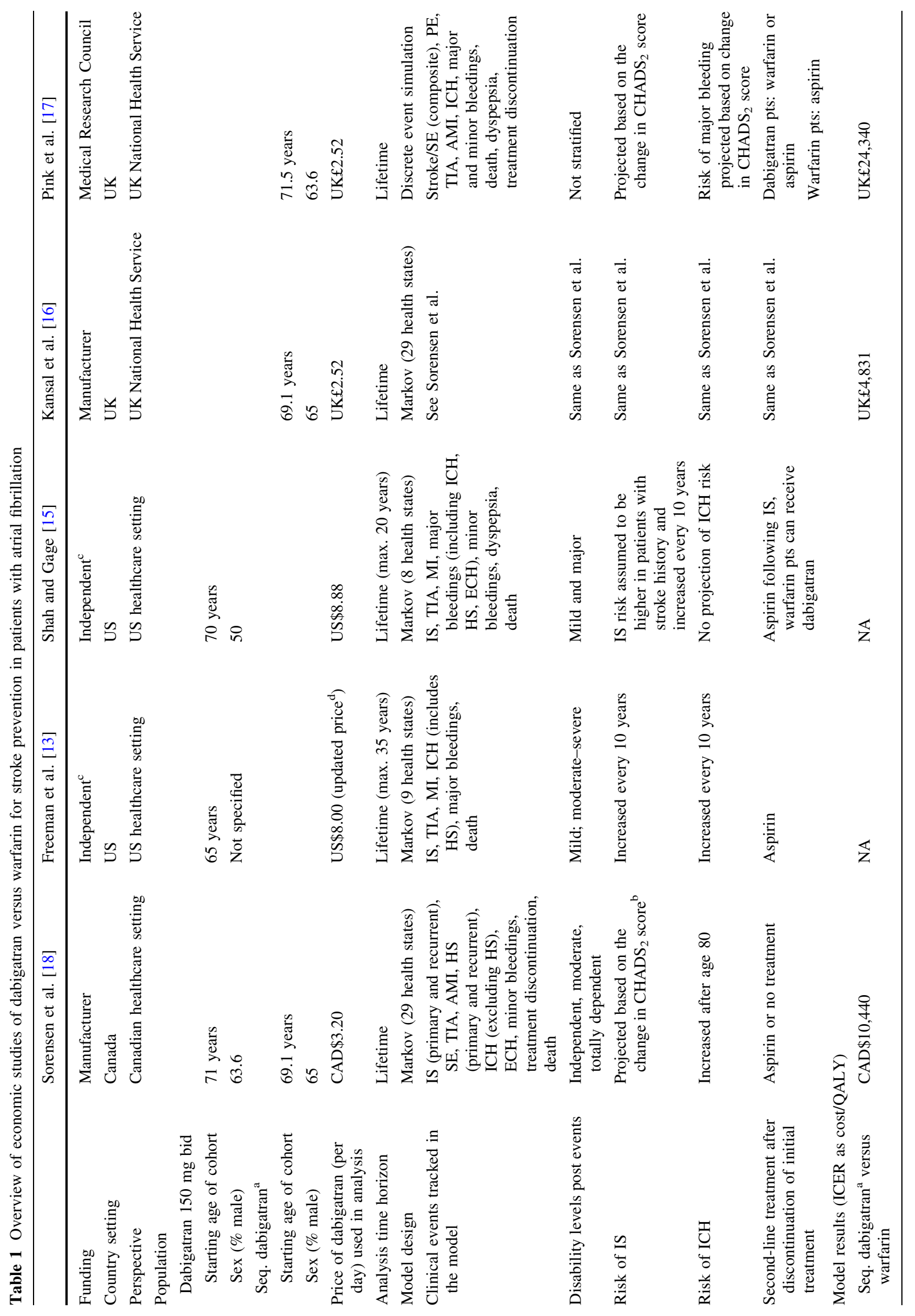




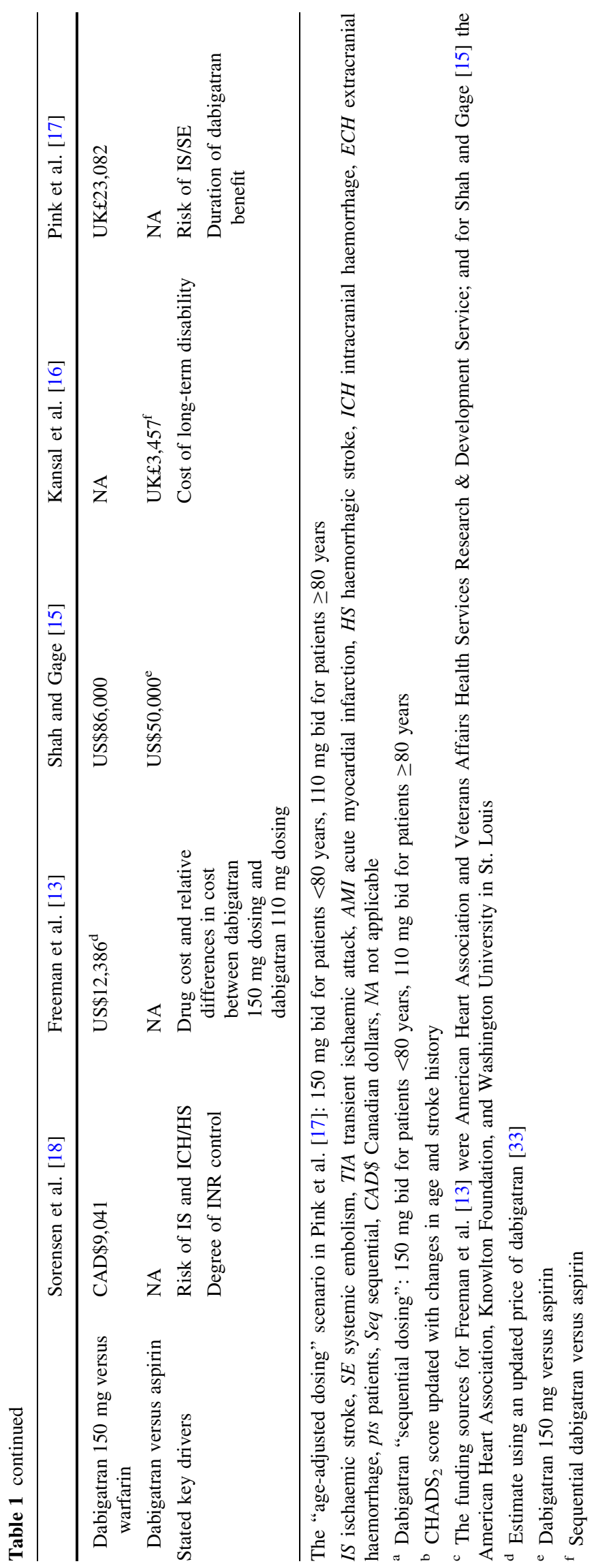


A detailed review of the selected cost-effectiveness analyses for dabigatran versus warfarin for stroke prevention in atrial fibrillation patients was undertaken in which the following aspects of the studies were extracted and evaluated: the modelling approach, the definition of the patient populations and their characteristics at baseline, comparators considered, clinical events probabilities, treatment effectiveness, adjustments to project future risk of stroke and bleeding events, cost and utility inputs, and drug switching and discontinuation.

The model previously published by us $[16,18]$ served as the reference model for comparisons of studies in the US and UK settings as this study required full access and flexibility to update the reference model programming to suit the comparison models. Sorensen et al. [18] presented results in the Canadian setting for dabigatran sequential use (the approved Canadian label), dabigatran $150 \mathrm{mg}$ bid (the approved US label), and dabigatran $110 \mathrm{mg}$ bid versus warfarin. This model was used as the reference model for the US setting because it has the relevant US dose included. In the Kansal et al. [16] model, sequential dosing for dabigatran (the approved UK label) was compared with trial-like warfarin, aspirin alone, and no treatment, from the UK healthcare perspective.

\subsection{Systematic Adaptation of Reference Model}

To identify the reasons for different results observed in models within the same setting, the reference model was adapted, as closely as possible, to use the inputs and key assumptions from each of the selected published models, including structural differences wherever possible. The objective was to determine if the reference model would produce results similar to the other model(s) once the cumulative effect of the different parameter values and assumptions were incorporated. The revisions to the reference model were conducted systematically. First the population characteristics (age and sex), model time horizon, discount rate, and stroke risk defined by $\mathrm{CHADS}_{2}$ score [cardiac failure, hypertension, age, diabetes, stroke (2 points)] at cohort start were changed. Second, all cost inputs for acute events, drugs, warfarin monitoring, and event follow-up were altered to match the model being compared. Finally, health-state utilities applied to acute events and resulting disability were altered. Clinical inputs and further model assumptions were also adapted as necessary. (See list of parameters in Tables 2, 3, 4.) Results were evaluated after each step. Analyses lower in the resulting tabulated findings reflect all changed parameters to this point (cumulative); incremental analyses identified those parameters with the greatest effects. The incremental costs, QALYs, and ICERs were compared after considering the cumulative effect of all the parameter value changes.
The reference model was implemented in Excel (version 2003).

\section{Overview of Studies}

\subsection{General Model Design}

The patient populations investigated in the published models were based on the RE-LY trial for patients with non-valvular AF, with and without a history of stroke, with only minor variations in starting patient age and sex distribution (Table 1). All studies used a Markov modelling approach, except Pink et al. [17], who used discrete event simulation (DES).

All models except one [16] presented results comparing dose-adjusted warfarin with dabigatran $150 \mathrm{mg}$ bid. The reference models and Pink et al. [17] also presented results for sequential dabigatran dosing $(150 \mathrm{mg}$ bid followed by $110 \mathrm{mg}$ bid). Additional comparators to dabigatran included aspirin [15, 16] or aspirin with clopidogrel [15].

The models accounted for second-line treatments triggered after clinical events or non-adherence. For patients on dabigatran, the second-line treatments were aspirin or warfarin [17] or aspirin only (reference models [13]). In the warfarin arm, aspirin was generally the second-line treatment option. An exception is the Shah and Gage model, which assigned warfarin patients to dabigatran following an ischaemic stroke [15]. This means that the comparator arm included dabigatran as a second-line treatment.

The models tracked the key clinical events recorded in the RE-LY trial. Only the reference models used post hoc analyses of the RE-LY patient-level data in addition to the published estimates to inform specific model parameters and to minimize the number of modelling assumptions. Additional post hoc analysis of the patient-level data was conducted in which the RE-LY population was stratified into those under 80 years of age and those at least 80 years of age. Pink et al. [17] used another post hoc analysis provided by Boehringer Ingelheim to the US Food and Drug Administration (FDA) [23] to inform the parameters for the comparison of warfarin versus sequential dabigatran.

The reference models tracked treatment status (on treatment, off treatment, switched), history of stroke, and level of disability (independent, moderate, and totally dependent) through the Markov health states. Pink et al. tracked treatment status, history of stroke, and level of disability within the DES approach [17]. The model diagrams by Freeman et al. [13] and Shah and Gage [15] did not indicate explicit tracking of stroke history and treatment status. All models followed patients for a lifetime, although "lifetime" was defined differently—a maximum 


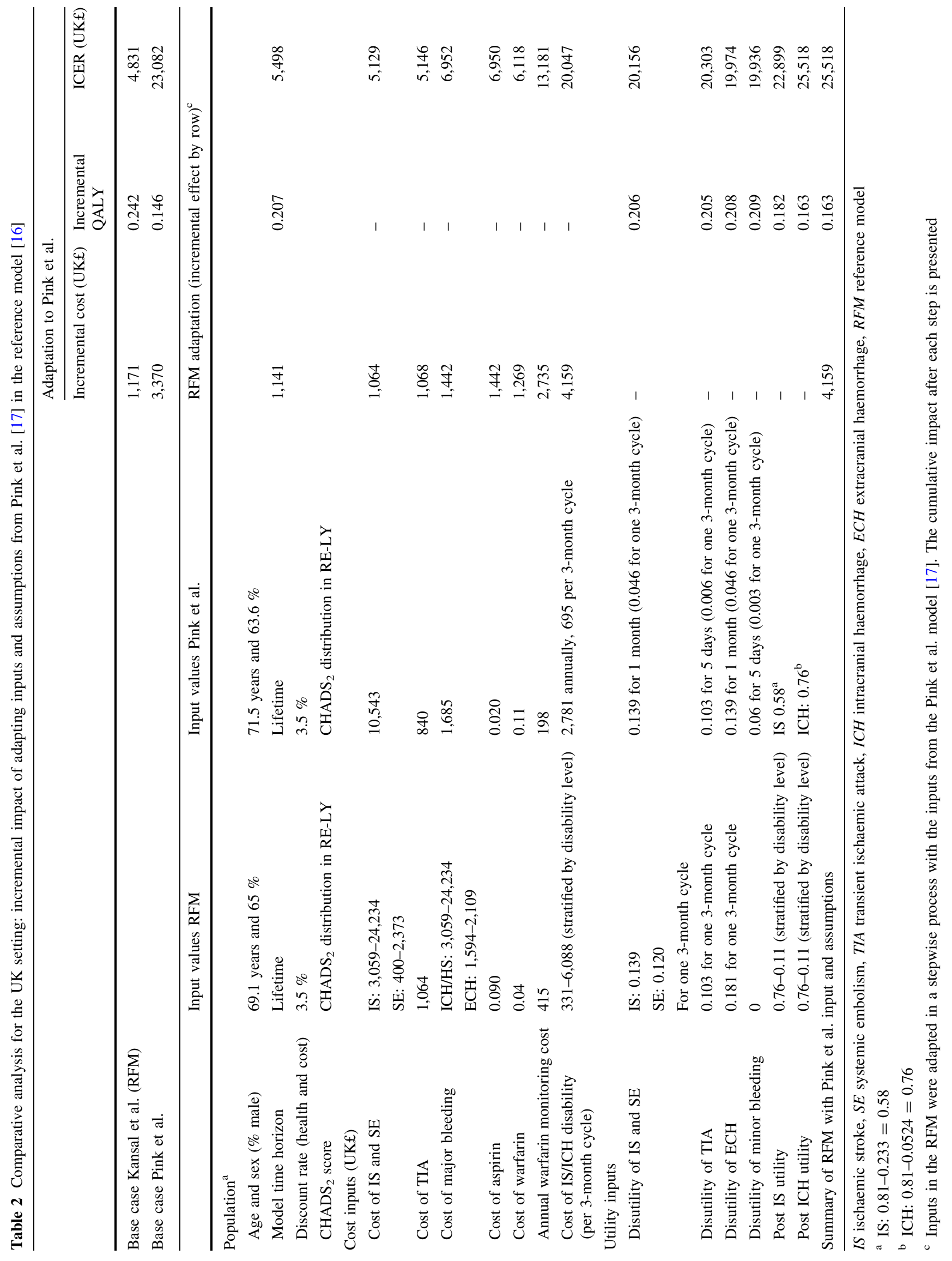




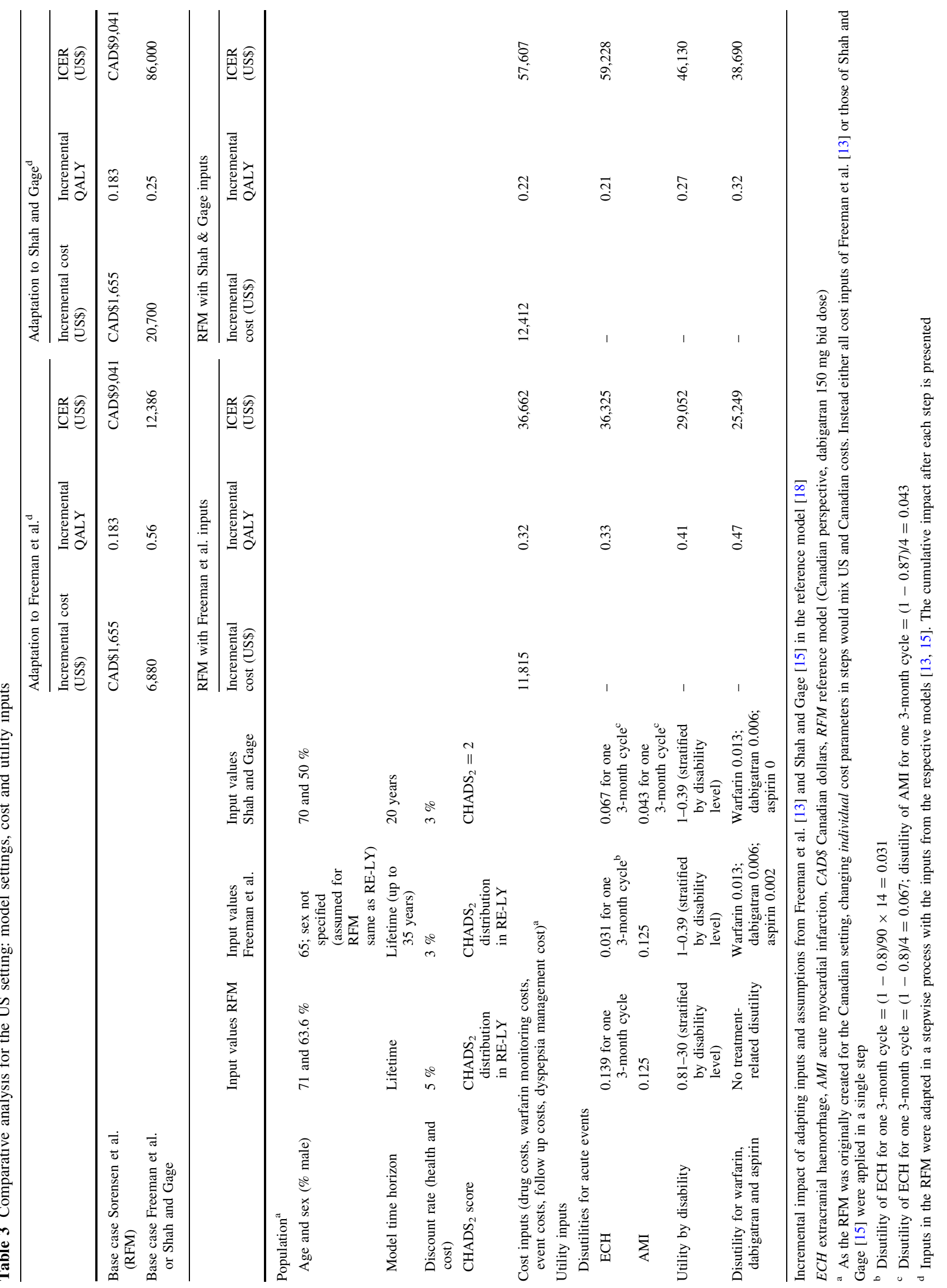




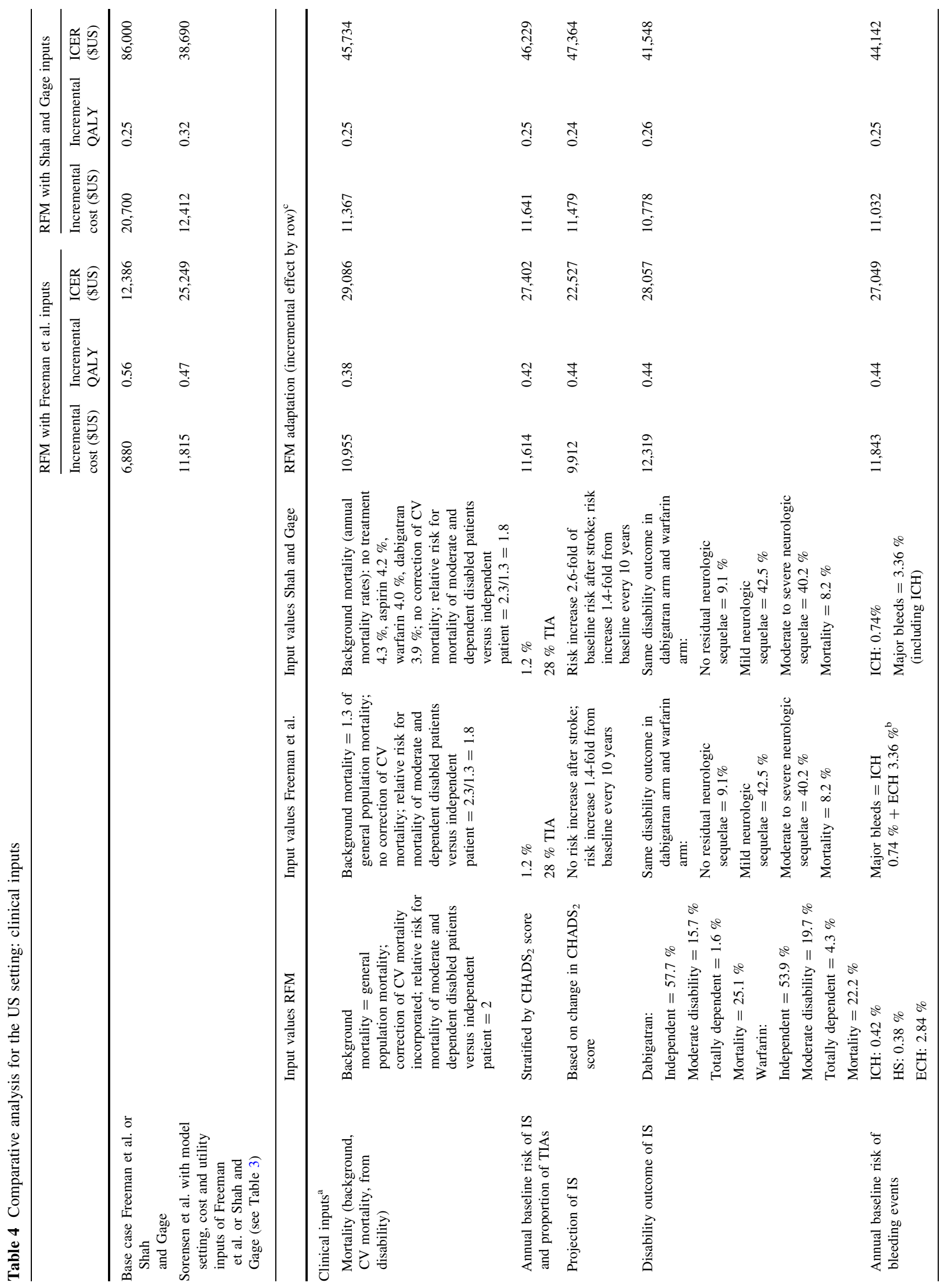




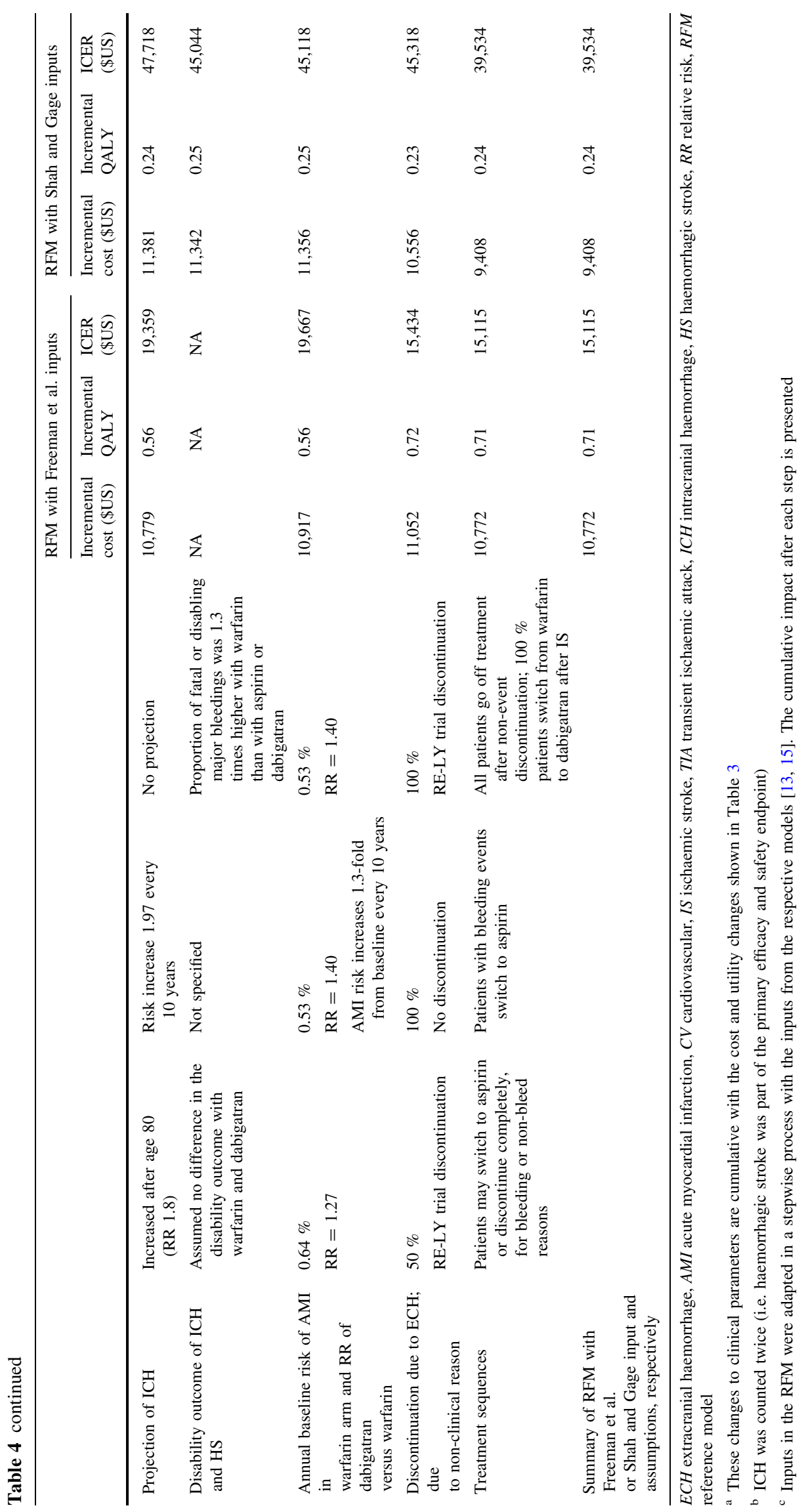


of 20 years in Shah and Gage [15], 35 years in Freeman et al. [13], up to age 100 in the reference models (roughly 31 years), and until death in Pink et al. [17].

The key clinical events, long-term disability from stroke and intracranial haemorrhage (ICH), and adjustments to risk of stroke and bleedings over the model time horizon are summarized in Table 1. Key differences between the models and the adaptations made to quantify their effect are summarized in Table 5.

\subsection{Risks of Events and Their Consequences}

The risk of clinical events and their consequences are key drivers of the cost-effectiveness results. In RE-LY, dabigatran $150 \mathrm{mg}$ bid demonstrated a lower risk of stroke and systemic embolism (SE) than warfarin [8,9], and in post hoc analyses dabigatran $150 \mathrm{mg}$ bid followed by dabigatran $110 \mathrm{mg}$ bid (sequential therapy) showed a similar pattern [18]. The incidence of major bleeding was generally similar in patients receiving dabigatran $150 \mathrm{mg}$ bid or warfarin, but the likelihood of ICH was less for both dabigatran doses $[8,9]$. As a consequence, inputs and assumptions of higher baseline risks for ICH events or more significant consequences of these events will result in greater benefits for dabigatran.

The risks of experiencing a stroke were generally similar across studies but were stratified differently. In the reference models, ischaemic stroke (IS) and haemorrhagic stroke (HS) were examined separately and IS risk was assigned according to $\mathrm{CHADS}_{2}$ score. In Pink et al. [17], the "stroke" endpoint estimates used in the model were those of the RE-LY primary composite endpoint (including IS, HS, and SE), and risk was based on $\mathrm{CHADS}_{2}$ scores. Freeman et al. and Shah and Gage used estimates aggregated across $\mathrm{CHADS}_{2}$ scores $[13,15]$. A composite risk of IS and transient ischaemic attack (TIA) was used in these models and allocated proportionally to IS or TIA. All models explored scenarios with different stroke risks to determine the most cost-effective population to treat.

The approach for adjusting the risk of stroke as patients aged and experienced events differed across the studies. In the reference models, the risk of stroke was dependent on $\mathrm{CHADS}_{2}$ score, which includes age and stroke history (higher score for age at least 75 years and previous stroke). Freeman et al. [13] assumed that the risks of stroke and TIA increase by a factor of 1.4 per decade of life; the same factor was used for both stroke-free and stroke-experienced patients. Shah and Gage [15] used the same estimate, but differentiated for patients with prior stroke (risk increase of 2.6).

The probabilities of major bleeding events captured the important safety profile of anticoagulants. In the reference models, the probabilities of ICH, HS, and extracranial major bleedings $(\mathrm{ECH})$ were estimated using patient-level data. The other models relied on estimates of major bleedings reported in the RE-LY trial that aggregated ICH and ECH. Pink et al. [17] stratified the risk of major bleedings by $\mathrm{CHADS}_{2}$ score. Freeman et al. appeared to count ICH twice, as the authors included major bleedings as defined in RE-LY (including ICH) and also considered ICH separately [13].

The adjustments for risk of bleeding over time also differed. In the reference models, the risk of ECH in patients under 70 years of age was half that of those over 70. For ICH in the reference models, the relative risk adjustment for patients over 80 years of age was 1.8 when a single dabigatran dose was evaluated. For the sequential dabigatran dosing analysis, both ICH and ECH risk changed at age 80. In Pink et al., the risk of non-HS bleedings remained constant over time, but was updated indirectly with age, as the risk of bleeding was assigned by $\mathrm{CHADS}_{2}$ score, which considers age [17]. Freeman et al. [13] assumed that the risk of ICH increased by 1.97 -fold per decade of life, whereas Shah and Gage [15] assumed no change in risk of bleeding over the 20-year model time horizon.

Stroke and ICH can result in long-term functional disability. In all models, quality of life (in the form of utilities) was adjusted on the basis of the severity of these clinical events. In the reference models, the consequences of stroke were categorized by the modified Rankin scale (mRS) and tracked as fatal, independent (mRS 0-2), moderately dependent (mRS 3-4), and totally dependent (mRS 5). This stratification was informed by a model-specific analysis of mRS scores recorded in the RE-LY study 3-6 months poststroke. The same disability categories were used to classify $\mathrm{ICH}$ on the basis of 3-month Glasgow Outcomes Scale (GOS) data from an observational study [24]. Both the Freeman et al. [13] and Shah and Gage [15] models classified the consequences of non-fatal strokes into three categories: no residual deficit, mild neurological residual deficit (results in neurologic deficit but no limitation in performing activities of daily living), and moderate to severe neurological residual deficit (results in loss of independence for at least one activity of daily living). In the Shah and Gage model [15], the severity of non-fatal ICH was not tracked. In Freeman et al. [13], ICH were classified into four categories: fatal, ICH with moderate to severe neurologic sequelae, ICH with mild neurologic sequelae, and ICH with no residual neurologic deficit. In Pink et al. [17], the proportion of non-fatal strokes that were non-disabling [defined as mRS $0-2(45 \%)$ ] or disabling (mRS 3-5) came from the RE-LY study [9] and stroke deaths reported in the FDA document [23]. Pink et al. [17] did not report any explicit consequences of ICH on mortality. 
Background mortality due to causes other than modelled clinical events was obtained from the general population in the reference models and Pink et al. [16-18]. In Freeman et al., the general population mortality was adjusted for non-valvular AF status and stroke history; however, death due to vascular events modelled such as stroke was not reported to be excluded from the general population mortality [13]. Shah and Gage assumed the background mortality rates differed by treatment groups $(4.0 \%$ for warfarin, and $3.9 \%$ for dabigatran both doses) and increased as the population aged [15].

\subsection{Treatment Status}

In all models, changes in treatment status were based on clinical events: major bleeding (all models), stroke [15], and non-adherence (reference models [15, 17]).

In the reference models, patients who experienced an ICH or HS permanently discontinued treatment; those who experienced an ECH continued the same treatment, temporarily stopped treatment, or discontinued any treatment permanently. Pink et al. [17], Shah and Gage [15], and Freeman et al. [13] assumed that patients who discontinued dabigatran or warfarin because of bleeding switched to aspirin. In Shah and Gage [15], patients who experienced both a stroke and a bleeding event were returned to their initial therapy.

The reference models and Freeman et al. did not consider switches for patient following an IS. In Shah and Gage [15], following an IS, all patients were given dabigatran $150 \mathrm{mg}$ bid, regardless of initial therapy. In Pink et al. [17], patients discontinuing dabigatran for nonbleeding reasons, which may have included IS, switched to warfarin, whereas patients discontinuing warfarin switched to aspirin.

The reference models considered discontinuation due to non-adherence and assumed a majority of patients would switch to aspirin. Pink et al. [17] considered discontinuation from non-bleeding reasons, which included nonadherence, as described above.

The proportion of patients discontinuing for non-event reasons differed among the models. In the reference models, Weibull functions were developed to project the probability of discontinuation for warfarin and dabigatran for up to 6 years. Pink et al. [17] used the 1- and 2-year discontinuation rates observed in RE-LY; thereafter, the discontinuation rate was assumed to be constant at the 2-year rate. In Shah and Gage [15], discontinuation for non-bleeding reasons affected drug costs, but not event rates; patients were assumed to continue receiving the treatment benefit after discontinuation for non-bleeding reasons.

\subsection{International Normalized Ratio Control}

INR control affects the number of events experienced by warfarin patients. If INR is too low, patients are more likely to experience ischaemic events, and if too high, patients are more likely to experience bleeding events. All models assumed that INR control reflected that of RE-LY (INR between 2.0 and 3.0 for an average of $64 \%$ of the time) in their base case analyses. All models except Freeman et al. [13] assessed the impact of alternative INR control scenarios on model results in sensitivity analysis. The reference models modified event risks in patients above or below the therapeutic INR range based on a study by Walker et al. [25], which reported the pattern of anticoagulant use and incidences of stroke, ICH, and SE in 116,969 AF patients. Shah and Gage [15] and Pink et al. [17] utilized a secondary analysis of the RE-LY data [26] that evaluated the efficacy and safety of dabigatran versus warfarin based on quartiles of INR control.

\subsection{Costs}

The cost per day of dabigatran is presented in Table 1 . Warfarin was relatively inexpensive in all models, but the annual costs for monitoring INR levels varied, with a higher cost being more favourable to dabigatran's costeffectiveness. The annual INR monitoring costs in Kansal et al. [16] [UK£414, based on an analysis undertaken by the National Institute for Health and Clinical Excellence (NICE)] [5] are more than twice that of Pink et al. [17] (UK£198, based on a micro-costing study). In the US setting, the cost for annual monitoring varied even more; the cost used by Shah and Gage was US $\$ 364$, whereas the cost used by Freeman et al. was US\$84 [13, 15].

Costs for acute management of events were applied at the time an event occurred. In the reference models, Freeman et al. and Shah and Gage, a follow-up cost was applied to each model cycle on the basis of the patient disability levels (reference models) or severity of the event defining the given health state [13, 15]. In Pink et al. [17], long-term costs were applied only to stroke (including SE) and MI, but not to bleeding events (such as ICH); major bleeding events, including both ICH and ECH, incurred an acute event cost similar to the ECH cost in Kansal et al. [16] (UK£1,685 in Pink et al. for ICH and ECH versus UK£1,594 for ECH in Kansal et al.). In contrast, the ICH events in Kansal et al. [16] incurred costs equivalent to those of HS and IS, reflecting the clinical severity of such events (ICH and HS costs ranging from UK£3,401 for independent disability to UK£24,234 for totally dependent disability). 
Table 5 Summary of key differences between models, including all identified published differences not tested in the reference model

\begin{tabular}{|c|c|c|c|}
\hline & Reference model & Comparison model & Reference model adaptation \\
\hline \multicolumn{4}{|l|}{ UK setting versus Pink et al. [17] } \\
\hline Model type & Markov cohort & Discrete event simulation & $\begin{array}{l}\text { Reference model not } \\
\text { adaptable }\end{array}$ \\
\hline Treatment effect on utility & No treatment disutility & $\begin{array}{l}0.013 \text { disutility for warfarin; } 0.002 \text { for } \\
\text { dabigatran }\end{array}$ & $\begin{array}{l}\text { Treatment disutility } \\
\text { incorporated }\end{array}$ \\
\hline Handling of ICH & $\begin{array}{l}\text { Separate event, similar to IS in } \\
\text { severity }\end{array}$ & $\begin{array}{l}\text { Aggregated with ECH as major } \\
\text { bleeding, much lower cost and } \\
\text { disutility than IS }\end{array}$ & $\begin{array}{l}\text { Cost and utility parameters } \\
\text { adapted }\end{array}$ \\
\hline Handling of MI & $\begin{array}{l}\text { No long-term consequences except } \\
\text { acute mortality risk }\end{array}$ & $\begin{array}{l}\text { Permanent disutility and cost post- } \\
\text { event }\end{array}$ & $\begin{array}{l}\text { Reference model not } \\
\text { adaptable }\end{array}$ \\
\hline \multicolumn{4}{|c|}{ US setting versus Freeman et al. [13] } \\
\hline Utility values & Healthy (with AF utility): 0.81 & Healthy (with AF) utility: 0.99 & Healthy state utility adapted \\
\hline Risk of IS and TIA over time & $\begin{array}{l}\text { Based on } \mathrm{CHADS}_{2} \text { score, } \\
\text { increases at age } 75 \text { and post-IS }\end{array}$ & $\begin{array}{l}\text { 1.4-fold increase in risk per decade of } \\
\text { life, no direct effect of stroke history }\end{array}$ & $\begin{array}{l}\text { Constant annual increase in } \\
\text { risk programmed }\end{array}$ \\
\hline Risk of ICH over time & Increased twofold at age 80 & 1.97-fold increase per decade of life & $\begin{array}{l}\text { Constant annual increase in } \\
\text { risk programmed }\end{array}$ \\
\hline Disability after second IS & $\begin{array}{l}\text { Assumed to be equal or worse than } \\
\text { pre-IS disability }\end{array}$ & $\begin{array}{l}\text { Moderate disability after two mild IS; } \\
\text { second moderate to severe IS fatal }\end{array}$ & $\begin{array}{l}\text { Reference model not } \\
\text { adaptable }\end{array}$ \\
\hline Disability after haemorrhage & $\begin{array}{l}\text { Distribution of permanent } \\
\text { disability post-ICH; no } \\
\text { permanent disability post-ECH }\end{array}$ & $\begin{array}{l}\text { Distribution of disability based on } \\
\text { ICH and ECH history }\end{array}$ & $\begin{array}{l}\text { Insufficient information on } \\
\text { distribution to replicate }\end{array}$ \\
\hline Treatment discontinuation & $\begin{array}{l}\text { Due to bleeding events or non- } \\
\text { adherence }\end{array}$ & Due to bleeding events only & $\begin{array}{l}\text { Discontinuation for non- } \\
\text { adherence disabled }\end{array}$ \\
\hline \multicolumn{4}{|c|}{ US setting versus Shah and Gage [15] } \\
\hline $\begin{array}{l}\text { Cost of post-IS and post-ICH } \\
\text { state }\end{array}$ & $\begin{array}{l}\text { Cost based on disability level } \\
\text { independent of event history }\end{array}$ & $\begin{array}{l}\text { Higher cost for patients experiencing } \\
\text { both ICH and IS events }\end{array}$ & $\begin{array}{l}\text { Reference model not } \\
\text { adaptable }\end{array}$ \\
\hline Utility values & Healthy (with AF utility): 0.81 & Healthy (with AF) utility: 0.99 & Healthy state utility adapted \\
\hline Risk of IS & $\begin{array}{l}\text { Based on } \mathrm{CHADS}_{2} \text { score, } \\
\text { increases at age } 75 \text { and post-IS }\end{array}$ & $\begin{array}{l}\text { 1.4-fold increase in risk per decade of } \\
\text { life, } 2.6 \text {-fold increase after first } \\
\text { stroke }\end{array}$ & $\begin{array}{l}\text { Annual increase in risk and } \\
\text { relative risk post-IS } \\
\text { programmed }\end{array}$ \\
\hline Risk of bleeding over time & $\begin{array}{l}\text { ICH increased twofold at age 80; } \\
\text { ECH at age } 70\end{array}$ & No change over time & $\begin{array}{l}\text { Risk of ECH and ICH set } \\
\text { constant }\end{array}$ \\
\hline Background rate of mortality & $\begin{array}{l}\text { General population mortality, } \\
\text { corrected for CV mortality }\end{array}$ & $\begin{array}{l}\text { Constant mortality rate stratified by } \\
\text { treatment arm }\end{array}$ & $\begin{array}{l}\text { Constant background } \\
\text { mortality adapted }\end{array}$ \\
\hline Treatment switching & $\begin{array}{l}\text { Patients switch from first-line } \\
\text { treatment to aspirin }\end{array}$ & $\begin{array}{l}\text { Patients switch to aspirin, dabigatran } \\
\text { (post-IS), or back to first-line } \\
\text { treatment (post-IS and ICH) }\end{array}$ & $\begin{array}{l}\text { Switching to dabigatran } \\
\text { post-IS programmed; } \\
\text { reference model unable to } \\
\text { be adapted to test switch } \\
\text { back to first-line treatment } \\
\text { after IS and ICH }\end{array}$ \\
\hline
\end{tabular}

$A F$ atrial fibrillation, $E C H$ extracranial haemorrhage, $M I$ myocardial infarction, $C V$ cardiovascular, $I S$ ischaemic stroke, $T I A$ transient ischaemic attack, $I C H$ intracranial haemorrhage

\subsection{Utilities}

Models assigned a baseline utility to the event-free state and disutilities for events experienced. In the reference models, the utility value of the "healthy" ("independent" disability health state), the health state in which patients spend the greatest proportion of their time, was 0.81; the corresponding baseline value was 0.774 in Pink et al. [17]. Both Freeman et al. [13] and Shah and Gage [15] applied utilities that differed according to treatment: 0.987 for healthy on warfarin and 0.994 for healthy on dabigatran. Similarly, Pink et al. [17] applied a disutility of 0.013 for patients on warfarin and a disutility of 0.002 for patients on dabigatran. The reference models did not apply a different utility according to treatment.

Post-event utility was stratified by disability level in the reference models, ranging from 0.65 ( 0.16 less than the "healthy" health state) to 0.30 (0.51 less than the "healthy" health state). In Pink et al. [17], the post-event disutility was defined by events, ranging from 0.0409 for MI 
to 0.233 for IS. It is important to note that ICH, which can result in more disability than stroke [27], was assigned a permanent disutility of only 0.0524 versus a permanent disutility of 0.233 for stroke. Pink et al. [17] also assigned a permanent impact on utility due to MI, in contrast to the other models, which assigned permanent impacts only to stroke and ICH. In the Freeman et al. model, the post stroke and ICH utilities were stratified as mild residual neurologic sequelae $(0.75)$ or moderate to severe residual neurologic sequelae (0.39). Permanent utility was assigned to MI at 0.84 [13]. In the Shah and Gage [15] model, post stroke and ICH utilities were stratified as mild residua, moderate to severe residua, and recurrent (range $0.12-0.75$ ). Temporary utilities were assigned to major bleeding other than $\mathrm{ICH}$, minor bleeding, and MI.

\section{Results: Comparative Analysis}

\subsection{UK Setting}

The initial comparison of the two models in the UK setting for the recommended dosing regimen of dabigatran resulted in incremental QALYs (dabigatran versus warfarin) of 0.146 in Pink et al. [17], and 0.242 in the reference model (Kansal et al. [16]), with a cost difference of UK£3,370 in Pink et al., and UK£1,171 in the reference model. Table 2 presents the cumulative impact of changing each cost and utility parameter in the reference model to reflect the values used in Pink et al. [17]. The reference model with population, cost, and utility inputs from Pink et al. [17] produced an incremental cost of UK£4,159 and incremental QALYs of 0.163 between the treatment arms. The resulting ICER, UK£25,518, was within $10 \%$ of the value reported by Pink et al. (UK£23,082), without changing any other clinical parameters [17].

The key reasons for differences between the two models were assumptions regarding long-term costs following intracranial events, costs of warfarin monitoring, and utility for ICH. Applying the long-term cost assumptions from Pink et al. [17] to the reference model increased the incremental cost from UK£2,735 in the previous step to UK£4,159 and brought the ICER to UK£20,047. The second most influential cost input to the ICER was the warfarin monitoring cost. Applying the monitoring cost from Pink et al. [17] to the reference model led to a doubling of the incremental cost of dabigatran from the previous step (UK£1,269 to UK£2,735) and increased the ICER from UK£6,118 to UK£13,181. Finally, Pink et al. [17] assigned a comparatively low permanent disutility to ICH. Changing the disutilities of IS and ICH in the reference model gave a $28 \%$ increase in the ICERs from UK£19,936 to UK£25,518.
Inputs that did not explain differences between the models were the acute event costs and disutilities assigned to IS and SE, TIA, and major bleeding, as well as drug costs.

\subsection{US Setting}

When the cost inputs and patient characteristics from Freeman et al. were placed into the reference model for adaptation to the US setting, the estimated incremental cost of dabigatran versus warfarin was $70 \%$ higher than the Freeman et al. results (reference model US $\$ 11,815$ versus Freeman et al. US\$6,880) (Tables 3, 4) [13]. Among the utility input changes, the greatest impacts were from the utilities characterizing post-event disability levels and utilities specific to treatments. These cumulative changes brought the incremental QALYs to 0.47 versus the incremental difference of 0.56 QALYs in Freeman et al. The population, cost, and utility adjustments resulted in an ICER about two times higher than reported in Freeman et al. [13], (reference model US\$25,249 versus Freeman et al. US\$12,386; Tables 3, 4).

An additional parameter that affected the findings to a large extent was the increased ICH risk with age. Applying the Freeman et al. [13] assumption in the reference model increased the incremental QALYs between dabigatran and warfarin, whereas the incremental costs remained largely unchanged. This assumption decreased the ICER estimated by the reference model to US $\$ 19,359$. Lastly, modifying the treatment discontinuation rates according to Freeman et al. [13] again affected mainly the QALY results rather than cost results. With all the adjustments, the reference model resulted in an ICER within $22 \%$ of the Freeman et al. [13] ICER (US\$15,115 versus US\$12,386).

Following a similar approach with the Shah and Gage model (i.e. adapting the reference model to the US setting by using the Shah and Gage patient characteristics and costs) resulted in an ICER of US $\$ 57,607$ per QALY in the reference model versus the reported ICER of US $\$ 86,000$ per QALY in Shah and Gage (Tables 3, 4) [15]. Updating the utility values in the reference model resulted in ICERs being further apart rather than closer together. The incremental QALYs after this step were 0.32 in the reference model versus 0.25 in Shah and Gage [15]. Applying the Shah and Gage mortality assumptions to the reference model aligned the incremental QALYs between the models [15]. Other revisions to clinical inputs made very small changes to these QALY results. With all adjustments, the reference model resulted in QALY estimates close to the Shah and Gage results, with incremental QALYs of 0.24 and 0.25 , respectively [15].

After adjusting costs in the reference model, incorporating the assumptions of Shah and Gage [15] regarding the 
projections of stroke and ICH risks over time, the resulting disability outcomes and the annual risk of AMI, only minimally changed the calculated incremental costs between dabigatran and warfarin. Assuming that warfarin patients received dabigatran following IS slightly decreased the difference in total costs between the two treatment arms. With all adjustments in the reference model, the incremental costs between dabigatran and warfarin remained below US $\$ 10,000$, less than half that in the Shah and Gage publication (reference model US $\$ 9,408$; Shah and Gage US\$20,700) [15]. Correspondingly, the final ICER is more than $50 \%$ lower than that reported by Shah and Gage (reference model US\$39,534; Shah and Gage US\$86,000) [15]. It was not possible to determine the reason(s) for the difference in incremental cost as all differences identified in the published model were applied to the reference model. In particular, the total incremental cost of the dabigatran population (including drug, monitoring, and acute and long-term costs following events) reported by Shah and Gage [15] exceeded the incremental cost of drug and monitoring alone in the adapted reference model (US\$20,700 versus US $\$ 15,176$, respectively) and all other cost differences (most acute and long-term costs following events) favoured dabigatran. In an effort to determine the source of the discrepant results, we performed simplified algebraic checks of the incremental drug and monitoring costs over a patient's remaining lifetime (9.1 years based on the mean discounted survival from the reference model). For example, an incremental cost of US $\$ 2,690 /$ year for dabigatran versus warfarin (i.e. its drug cost and associated monitoring) and conservatively assuming that $20 \%$ of patients discontinue treatment over the first 2 years, as reported by Shah and Gage [15], but the remaining $80 \%$ of patients remain adherent for the rest of their lives, implies a maximum incremental drug and monitoring cost of US\$20,120, with any additional discontinuations or reduction in clinical events with dabigatran reducing this value. This means that the total incremental cost of the dabigatran arm in the adapted reference model will always be less than US $\$ 20,120$ and thus cannot match the higher value found by Shah and Gage [15].

\section{Discussion}

Given the publication of a number of studies examining the cost-effectiveness of dabigatran versus warfarin for stroke prevention in patients with $\mathrm{AF}$, it was possible to conduct a quantitative comparison of the economic models using a reference model approach. The model previously published by us $[16,18]$ served as the reference for comparisons of studies in the US and UK settings. The reference model was adapted using the inputs and key assumptions from each of the other published studies to determine if results could be reproduced, providing a more accurate and precise understanding of the impact of specific model assumptions than could be achieved with a qualitative review and descriptive comparison of published models. This enables a better interpretation of published findings by focusing attention on the assumptions underlying the key model features accounting for differences among model results.

Recently, several qualitative reviews of cost-effectiveness studies of dabigatran have been published [28-30]. Reddy et al. [29] presented a review of the clinical and economic evidence for dabigatran in patient with $\mathrm{AF}$ and for the prevention of venous thromboembolism. Of the models considered in the present study, only Freeman et al. [13] was mentioned in the review by Reddy et al. [29]. The review conducted by Solomon and colleagues included study results from Freeman et al. [13], Shah and Gage [15], Sorensen et al. [18], Pink et al. [17], and the UK ERGreviewed model (based on Kansal et al. [16]) [5], with a broad goal of providing guidance to policy makers and clinicians when making decisions about the optimal stroke prophylaxis strategy in atrial fibrillation patients [30]. The authors acknowledged the challenges regarding the qualityof-life data and the uncertainty of long-term care costs, consistent with the important effect of these parameters in this review. McKeage et al. [28] also presented a detailed summary of the findings of eight models [5, 13-19]. The author cited some limitations of the studies including the applicability of RE-LY data to various populations. These previously published reviews, however, do not critically appraise the models or explain reasons for differences in the ICER results. Our study sought to quantify the impact of specific differences between the studies on the ICER results. Not all features of the comparison models could be implemented in the reference model, however, such as the assumption of long-term consequences of MI in Pink et al., and the specialized handling of those patients experiencing both IS and ICH events in Shah and Gage [15, 17]. These unaccounted differences (tabulated in Table 5) are limitations of this analysis. As discussed below, however, in general the adaptation process was able to closely replicate most of the cost and QALY results of the comparison models.

Our study found that the differences in reported costeffectiveness results across the models examined can, in general, be explained on the basis of assumptions and parameter values reported in the studies. In the UK setting, the differences in model results could be largely explained by cost and utility model inputs (Table 2). The assumed cost and quality of life following ICH events and warfarin monitoring costs accounted for nearly all of the difference in reported results between the reference model (Kansal 
et al. [16]) and Pink et al. model [17]. Modelling of ICH differed notably in Pink et al. [17] from the other published models. In particular, the other models, including the reference model, assigned acute and permanent cost and quality-of-life detriments to ICH that were similar to, or greater than, those assigned to IS. In contrast, in Pink et al. [17], the acute utility impact from an $\mathrm{ICH}$ was valued at 0.0524 , less than one-quarter of the corresponding value for stroke $(0.233)$, and cost of ICH events was not assigned separately but aggregated with $\mathrm{ECH}$, yielding a relatively low cost. Additionally, there were no long-term costs postICH in the Pink et al. model, despite the clinical severity and long-term disability associated with these events [17]. Given the significant reduction in the rate of $\mathrm{ICH}$ with dabigatran relative to warfarin, the Pink et al. [17] approach to ICH underestimates dabigatran's cost-effectiveness relative to the assumption made in the other models reviewed.

In the US setting, there were important differences between the models that went beyond unit cost and utility values. The most important variables distinguishing results in Freeman et al. from those of the reference model were the adjustments to the risk of ICH and IS as the modelled cohorts aged [13]. Although Freeman et al. assumed a continuous increase in the risk of IS and ICH with age, the magnitude of this increase over a 35-year time horizon may be difficult to justify [13]. In addition, Freeman and colleagues appear to count the risk of ICH twice (as a separate event and as part of major bleeding) [13]. Given the reduction in IS and $\mathrm{ICH}$ rates for dabigatran, the increases in baseline event risks tend to favour dabigatran in the costeffectiveness, as does the fact that discontinuation due to non-adherence was not included in the model. There was also a notable difference in the baseline utility of patients in the models, which can substantially alter the predicted value of preventing a stroke.

Although the reference model could reasonably replicate final ICER results for the other models, it could not replicate the results of Shah and Gage [15] in the US setting. QALY results could be replicated quite closely, with the most important adaptations being the use of the utility values and mortality assumptions from Shah and Gage [15]. The cost results, however, could not be replicated, as the total incremental cost of dabigatran published by Shah and Gage exceeded the incremental cost of drug and monitoring in the adapted reference model and all other cost differences favoured dabigatran [15]. The final incremental cost, and thus the final ICER, in the reference model is therefore less than half that reported by Shah and Gage [15]. The only identified published model characteristics not replicated in the adapted reference model were the treatment choice and costs of patients who experience both IS and ICH, but these are expected to be small contributors as very few patients experience both events and their survival should be relative short.

\section{Conclusions}

Overall, this comparison found that the differences for the cost-effectiveness of dabigatran versus warfarin among published models could be reasonably well explained by differences in reported parameters and assumptions. Important differences found were those related to the patients' risk of having intracranial events, treatment discontinuation, and mortality rates. In addition, incremental costs were highly sensitive to differences in long-term cost of disability and warfarin monitoring. Similarly, differences in the utility values following events with long-term disability affected incremental QALYs.

As additional new oral anticoagulants become available, it will be important to assess how they compare to one another [31, 32] and how robust those analyses are across studies. This study highlighted a few key assumptions that impact the overall cost-effectiveness estimates of dabigatran versus warfarin. As noted above, the costs of follow-up care and health-related quality of life of patients who experience an $\mathrm{ICH}$ event have a strong impact on overall findings. A study to quantify these post-event consequences even more accurately may be warranted. As anticoagulation treatment is expected to be given for the remainder of AF patients' lives, improved understanding of the clinical risk in AF patients of IS and $\mathrm{ICH}$ over time may also allow better interpretation of cost-effectiveness analyses.

Despite the differences in modelling methods, assumptions, and parameter values, however, all of the modelling studies reviewed reached generally similar conclusions about the cost-effectiveness of dabigatran in their local settings. This is consistent with the final assessments by governmental health technology assessment bodies, including NICE and the Canadian Agency for Drugs and Technology in Health [5, 10].

Acknowledgments The project was funded by Boehringer Ingelheim International GmbH. All authors contributed to the study concept, data interpretation, and writing of the manuscript. In addition, Anuraag Kansal, Siyang Peng, and Sonja Sorensen contributed the model analyses. Sonja Sorensen, Siyang Peng, and Anuraag Kansal acted as paid consultants on this project. Brigitta U. Monz and Carole Bradley-Kennedy are employees of Boehringer Ingelheim, the manufacturer of dabigatran.

Open Access This article is distributed under the terms of the Creative Commons Attribution Noncommercial License which permits any noncommercial use, distribution, and reproduction in any medium, provided the original author(s) and the source are credited. The exclusive right to any commercial use of the article is with Springer. 


\section{Reference}

1. National Institute for Health and Clinical Excellence (NICE). Costing report: implementing NICE guidance in England. Atrial fibrillation: the management of atrial fibrillation. NICE clinical guideline 36. London: National Institute for Health and Clinical Excellence; 2006.

2. Go AS, Hylek EM, Phillips KA, et al. Prevalence of diagnosed atrial fibrillation in adults: national implications for rhythm management and stroke prevention: the AnTicoagulation and Risk Factors in Atrial Fibrillation (ATRIA) Study. JAMA. 2001;285(18):2370-5.

3. Luengo-Fernandez R, Yiin GS, Gray AM, et al. Population-based study of acute- and long-term care costs after stroke in patients with AF. Int J Stroke. 2012. doi:10.1111/j.1747-4949.2012.00812.x.

4. Roger VL, Go AS, Lloyd-Jones DM, et al. Heart disease and stroke statistics-2012 update: a report from the American Heart Association. Circulation. 2012;125(1):e2-220.

5. National Institute for Health and Clinical Excellence (NICE). Atrial fibrillation-dabigatran etexilate (TA249). Final guidance [NICE technology appraisal guidance no. 249; online]. London: National Institute for Health and Clinical Excellence; 2012. http://www.nice.org.uk/ta249. Accessed 19 Mar 2012.

6. Skanes AC, Healey JS, Cairns JA, et al. Focused 2012 update of the Canadian Cardiovascular Society atrial fibrillation guidelines: recommendations for stroke prevention and rate/rhythm control. Can J Cardiol. 2012;28(2):125-36.

7. Wann LS, Curtis AB, Ellenbogen KA, et al. 2011 ACCF/AHA/ HRS focused update on the management of patients with atrial fibrillation (update on dabigatran). A report of the American College of Cardiology Foundation/American Heart Association Task Force on Practice Guidelines. Heart Rhythm. 2011;8(3): e1-8.

8. Connolly SJ, Ezekowitz MD, Yusuf S, et al. Dabigatran versus warfarin in patients with atrial fibrillation. $\mathrm{N}$ Engl $\mathrm{J}$ Med. 2009;361(12):1139-51.

9. Connolly SJ, Ezekowitz MD, Yusuf S, et al. Newly identified events in the RE-LY trial. N Engl J Med. 2010;363(19):1875-6.

10. Canadian Agency for Drugs and Technology in Health (CADTH). CEDAC final recommendation: dabigatran etexilate. In: New indication: prevention of stroke and systemic embolism in patients with atrial fibrillation. Ottawa: Canadian Agency for Drugs and Technology in Health (CADTH).

11. Pharmaceutical Benefits Advisory Committee (PBAC). Public summary document: dabigatran etexilate. Department of Health and Ageing, Australian Government. Australia: Canberra; 2011.

12. Scottish Medicines Consortium. Dabigatran etexilate $110 \mathrm{mg}$ and $150 \mathrm{mg}$ hard capsules (Pradaxa ${ }^{\circledR}$ ). 12 September 2011. Report no.: SMC No. (672/11) Scotland: Glasgow.

13. Freeman JV, Zhu RP, Owens DK, et al. Cost-effectiveness of dabigatran compared with warfarin for stroke prevention in atrial fibrillation. Ann Intern Med. 2011;154(1):1-11.

14. Kamel H, Johnston SC, Easton JD, et al. Cost-effectiveness of dabigatran compared with warfarin for stroke prevention in patients with atrial fibrillation and prior stroke or transient ischemic attack. Stroke. 2012;43(3):881-3.

15. Shah SV, Gage BF. Cost-effectiveness of dabigatran for stroke prophylaxis in atrial fibrillation. Circulation. 2011;123(22): 2562-70.

16. Kansal AR, Sorensen SV, Gani R, et al. Cost-effectiveness of dabigatran etexilate for the prevention of stroke and systemic embolism in UK patients with atrial fibrillation. Heart. 2012;98(7):573-8.

17. Pink J, Lane S, Pirmohamed M, et al. Dabigatran etexilate versus warfarin in management of non-valvular atrial fibrillation in UK context: quantitative benefit-harm and economic analyses. BMJ. 2011;343:d6333.

18. Sorensen SV, Kansal AR, Connolly S, et al. Cost-effectiveness of dabigatran etexilate for the prevention of stroke and systemic embolism in atrial fibrillation: a Canadian payer perspective. Thromb Haemost. 2011;105(5):908-19.

19. Langkilde LK, Bergholdt Asmussen M, Overgaard M. Costeffectiveness of dabigatran etexilate for stroke prevention in nonvalvular atrial fibrillation. Applying RE-LY to clinical practice in Denmark. J Med Econ. 2012;15(4):695-703.

20. Braithwaite RS, Meltzer DO, King JT Jr, et al. What does the value of modern medicine say about the $\$ 50,000$ per qualityadjusted life-year decision rule? Med Care. 2008;46(4):349-56.

21. Devlin N, Parkin D. Does NICE have a cost-effectiveness threshold and what other factors influence its decisions? A binary choice analysis. Health Econ. 2004;13(5):437-52.

22. Clarke LD, Plevritis SK, Boer R, et al. A comparative review of CISNET breast models used to analyze U.S. breast cancer incidence and mortality trends. J Natl Cancer Inst Monogr. 2006;36: 96-105.

23. Boehringer Ingelheim Pharmaceuticals Inc. Advisory committee briefing document-dabigatran etexilate. Food and Drug Administration, 2010. http://www.fda.gov/downloads/advisory committees/committeesmeetingmaterials/drugs/cardiovascular andrenaldrugsadvisorycommittee/ucm226009.pdf). Accessed 18 April 2012.

24. Rosand J, Eckman MH, Knudsen KA, et al. The effect of warfarin and intensity of anticoagulation on outcome of intracerebral hemorrhage. Arch Intern Med. 2004;164(8):880-4.

25. Walker AM, Bennett D. Epidemiology and outcomes in patients with atrial fibrillation in the United States. Heart Rhythm. 2008;5(10):1365-72.

26. Wallentin L, Yusuf S, Ezekowitz MD, et al. Efficacy and safety of dabigatran compared with warfarin at different levels of international normalised ratio control for stroke prevention in atrial fibrillation: an analysis of the RE-LY trial. Lancet. 2010;376(9745):975-83.

27. Bamford J, Sandercock P, Dennis M, et al. A prospective study of acute cerebrovascular disease in the community: the Oxfordshire Community Stroke Project-1981-86. 2. Incidence, case fatality rates and overall outcome at one year of cerebral infarction, primary intracerebral and subarachnoid haemorrhage. J Neurol Neurosurg Psychiatry. 1990;53(1):16-22.

28. McKeage K. Dabigatran etexilate: a pharmacoeconomic review of its use in the prevention of stroke and systemic embolism in patients with atrial fibrillation. Pharmacoeconomics. 2012;30(9): 841-55.

29. Reddy P, Atay JK, Selbovitz LG, et al. Dabigatran: a review of clinical and pharmacoeconomic evidence. Crit Pathw Cardiol. 2011;10(3):117-27.

30. Solomon MD, Ullal AJ, Hoang DD, et al. Cost-effectiveness of pharmacologic and invasive therapies for stroke prophylaxis in atrial fibrillation. J Cardiovasc Med (Hagerstown). 2012;13(2): 86-96.

31. Canadian Agency for Drugs and Technology in Health (CAD$\mathrm{TH})$. New oral anticoagulants for the prevention of thromboembolic events in patients with atrial fibrillation. Ottawa: Canadian Agency for Drugs and Technology in Health (CADTH); 2012.

32. Kansal AR, Sharma M, Bradley-Kennedy C, et al. Dabigatran versus rivaroxaban for the prevention of stroke and systemic embolism in atrial fibrillation in Canada. Comparative efficacy and cost-effectiveness. Thromb Haemost. 2012;108(4):672-82.

33. Freeman J, Turakhia M. Dabigatran compared with warfarin for stroke prevention in atrial fibrillation (Author reply). Ann Intern Med. 2011;154(8):570-1. 